doi:10.1038/pr.2016.137

\section{Allergy and ADHD/ODD}

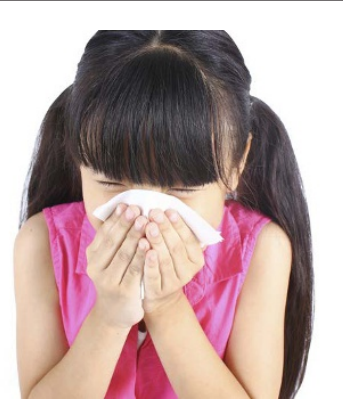

Lin and coauthors investigated the detailed associations between allergic diseases with attention deficit/ hyperactivity disorder (ADHD) and oppositional defiant disorder in children. Analysis of clinical information for nearly 3,000 children enrolled in the Taiwan Children Health Study appeared to show that children with symptoms of allergic diseases were more likely to develop different subtypes of ADHDrelated behavioral disorders.

See page 480

\section{Unexplained neonatal death or collapse}

There is currently no national system in

Australia for reporting and investigating cases of sudden unexpected early neonatal death or acute life-threatening events. In a three-year prospective national surveillance study of New South Wales and Queensland, Lutz et al. found the incidence of such events to be 0.1 and 0.08 in 1,000 live births, respectively. Among 48 definitive cases, 26 babies collapsed on day 1 , and 19 were found on the carer's chest. Development and implementation of guidelines for safe sleeping, in the hospital as well as in the community, are needed.

See page 493

\section{Hippocampal size and congenital heart disease}

Latal and coinvestigators sought to determine whether hippocampal volume was reduced in adolescents

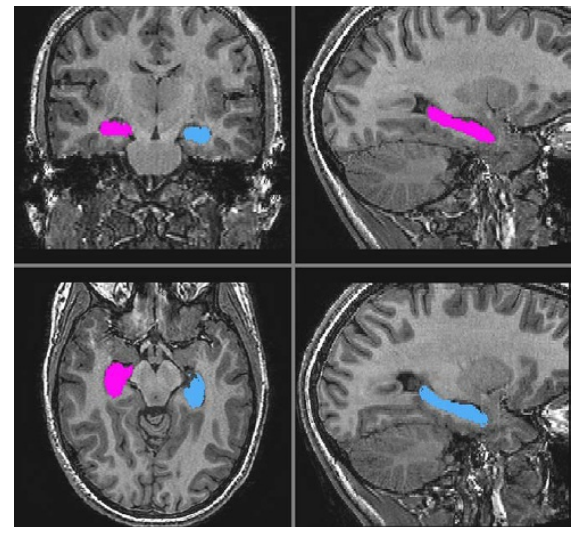

with congenital heart disease (CHD) and whether altered volumes were related to functional outcome. At a mean age of 13.8 years, 48 adolescent survivors of childhood cardiopulmonary bypass surgery for CHD and 32 healthy controls underwent neurocognitive testing and cerebral magnetic resonance imaging. The adolescents with CHD had $10 \%$ lower total hippocampal volumes compared with controls. See page 531

\section{CSF cytokines in infant meningitis}

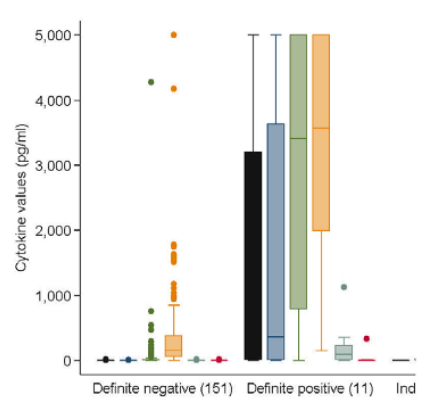

Pro- and anti-inflammatory cytokines are elevated in bacterial meningitis and may be useful diagnostic adjuncts when cerebrospinal fluid (CSF) cultures are negative. Srinivasan et al. used cytometric bead arrays to measure specific cytokines present in infant CSF. Receiver operating characteristic analyses and principal component analysis were used to determine cytokine combinations that identified bacterial meningitis. The authors found that interleukin- 6 and -10 were the individual cytokines that provided the greatest accuracy in diagnosing cultureproven bacterial meningitis.

See page 566

\section{miR-205 in obstructive nephropathy}

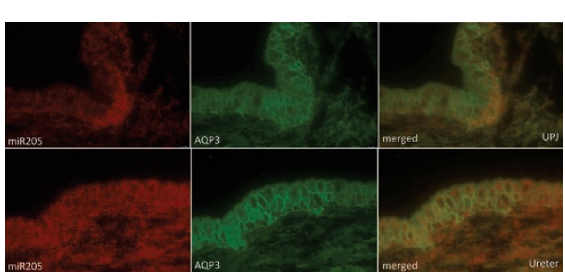

A better understanding of molecular and cellular changes is needed to facilitate development of improved biomarkers and novel therapeutic approaches in congenital obstructive nephropathy (CON). Wilhide and colleagues characterized a particular microRNA, miR-205, whose expression changes with the degree of hydronephrosis in the megabladder (mgb) mouse kidney. They found that miR-205 increased with severity of CON in the $\mathrm{mgb}^{-/-}$mouse and may regulate urothelial differentiation. See page 602

\section{Developmental gene regulation in ductus arteriosis}

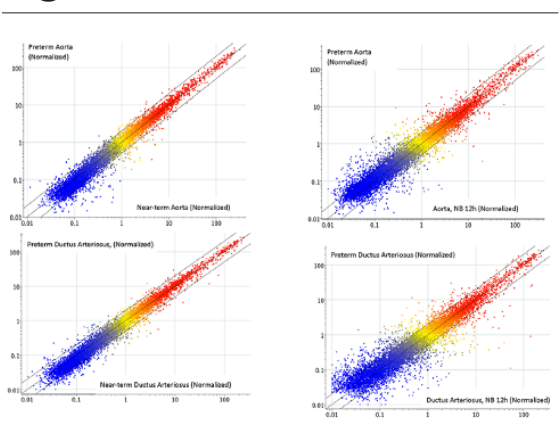

Goyal and co-researchers used fetal and neonatal sheep to test the hypothesis that development of ductus arteriosis (DA) is associated with significant alterations in specific mRNA expression. They conducted oligonucleotide microarray experiments on isolated $\mathrm{mRNA}$ from DA and ascending aorta from premature, near-term, and newborn lambs, and compared alterations in mRNA expression. Results identified several candidate genes that may be important in DA maturation. See page 610 